\title{
Plasmon Geometric Phase and Plasmon Hall Shift
}

\author{
Li-kun Shi ${ }^{1}$ and Justin C. W. Song ${ }^{1,2, *}$ \\ ${ }^{1}$ Institute of High Performance Computing, Agency for Science, Technology, and Research, \\ Singapore 138632 \\ ${ }^{2}$ Division of Physics and Applied Physics, Nanyang Technological University, Singapore 637371
}

(Received 6 November 2017; revised manuscript received 22 February 2018; published 17 April 2018)

\begin{abstract}
The collective plasmonic modes of a metal comprise a simple pattern of oscillating charge density that yields enhanced light-matter interaction. Here we unveil that beneath this familiar facade plasmons possess a hidden internal structure that fundamentally alters its dynamics. In particular, we find that metals with nonzero Hall conductivity host plasmons with an intricate current density configuration that sharply departs from that of ordinary zero Hall conductivity metals. This nontrivial internal structure dramatically enriches the dynamics of plasmon propagation, enabling plasmon wave packets to acquire geometric phases as they scatter. At boundaries, these phases accumulate allowing plasmon waves that reflect off to experience a nonreciprocal parallel shift. This plasmon Hall shift, tunable by Hall conductivity as well as plasmon wavelength, displaces the incident and reflected plasmon trajectories and can be readily probed by nearfield photonics techniques. Anomalous plasmon geometric phases dramatically enrich the nanophotonics toolbox, and yield radical new means for directing plasmonic beams.
\end{abstract}

DOI: 10.1103/PhysRevX.8.021020

Subject Areas: Condensed Matter Physics, Plasmonics

\section{INTRODUCTION}

The internal structure of quasiparticles [1-6], e.g., spin, valley degrees of freedom, while typically hidden from view can dramatically alter the behavior of particles when they are coupled with kinematic variables. A key ingredient for nontrivial band topology and associated gapless edge states in fermionic [1,2] and bosonic systems [7,8], this coupling also warps bulk band geometry allowing the bands to acquire nontrivial (pseudo)spin texture. This texture enriches the dynamics of bulk band quasiparticles, enabling them to acquire geometric phases that skew their trajectories, transforming their response to external fields [9-13].

In a metal, collective plasmon quasiparticles can emerge out of the self-sustained oscillations of charge density and electric field $\mathbf{E}$ degrees of freedom. For two-dimensional metals, $\mathbf{E}$ and charge density oscillate in lockstep allowing deep subwavelength plasmons to be excited [14-16]. These feature longitudinal electric (LE) modes (with field orientation and propagation direction aligned) that enable a range of nanophotonic properties far below the diffraction limit [16-19]. As such, intense efforts have focused on

\footnotetext{
*justinsong@ntu.edu.sg
}

Published by the American Physical Society under the terms of the Creative Commons Attribution 4.0 International license. Further distribution of this work must maintain attribution to the author(s) and the published article's title, journal citation, and DOI. unraveling its dispersion and exploiting its tightly bound electric fields [14-21].

Here we argue that, in addition to $\mathbf{E}$ and charge density fields, 2D deep subwavelength bulk plasmons can possess a finer internal structure (Fig. 1) that dramatically alters its dynamics. In particular, we find that plasmon current density configuration depends intimately on a metal's Hall conductivity $\sigma_{x y}$, exhibiting an intricate pattern that cants away from the propagation direction when $\sigma_{x y} \neq 0$ [Fig. 1(b)]. This pattern is characterized by a plasmon current density pseudospinor that tracks in-plane current density orientation and phase [Figs. 1(c) and 1(d)] and enables a striking microscopic distinction between plasmons in conventional $\sigma_{x y}=0$ metals and $\sigma_{x y} \neq 0$ metals. Plasmons in the latter possess a nontrivial hedgehoglike current density texture [Fig. 1(d)] hosted within the 2D metal.

Crucially, the nontrivial current density pseudospin texture directly affects the dynamics of plasmon waves, allowing them to accumulate geometric phases as they propagate. In particular, we demonstrate that for $\sigma_{x y} \neq 0$ plasmon plane waves acquire an additional geometric phase (directly tied to the nontrivial texture) when they scatter off a boundary. Strikingly, these phases accrue when plane waves are superposed, allowing wave packets that reflect off boundaries to experience a parallel translation along the boundary-plasmon Hall shift [Fig. 3] — displacing the incident and reflected plasmon beams by multiple plasmon wavelengths. As we show below, this plasmon Hall shift is geometrical and directly arises from a geometric connection between the incident and reflected waves. As a result, a 
(a)

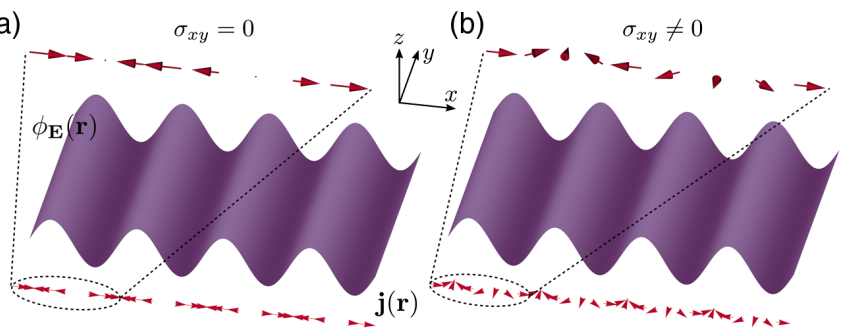

(c)

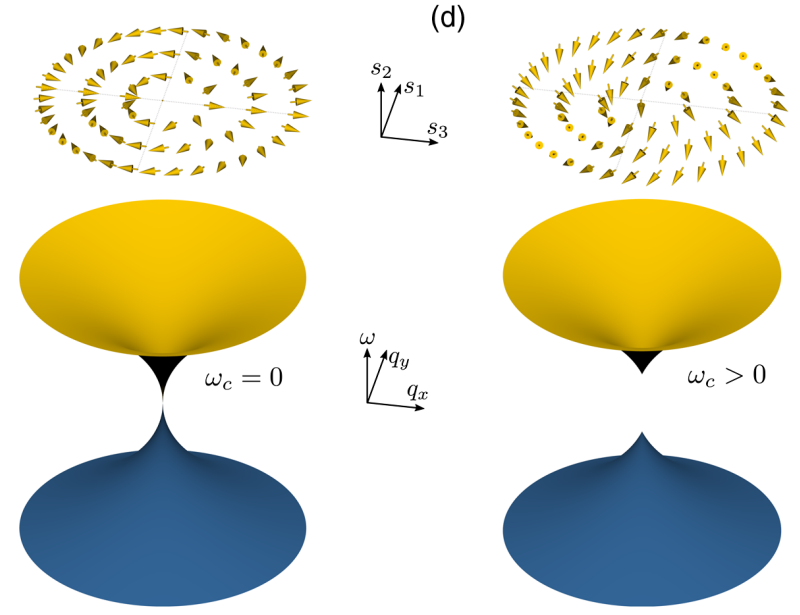

FIG. 1. Plasmon dispersion and internal current density texture. (a),(b) Current density (vector) fields $\mathbf{j}(\mathbf{r})$ configuration for plasmons with $\sigma_{x y}=0$ and $\sigma_{x y} \neq 0$, respectively. Note that while electric potential $\phi_{\mathbf{E}}(\mathbf{r})$ take on the same longitudinal electric profile, $\mathbf{j}(\mathbf{r})$ adopts a completely different texture. Plasmon dispersion for (c) conventional plasmon $\left(\omega_{c}=0\right)$, and (d) magnetoplasmon modes $\left(\omega_{c} \neq 0\right)$ in a two-dimensional metal [Eq. (5)], both in the nonretarded limit. Current density pseudospinor [Eq. (7)] for $\omega_{c}>0$ exhibits a nontrivial canted texture; when $\omega_{c}=0$, pseudospinor winds only in the $\left(s_{1}, s_{3}\right)$ plane. Blue band is a complex-conjugate copy of primary plasmon band (see text).

nonreciprocal plasmon Hall shift manifests even though plasmons are charge neutral, with sign controlled by the incident wave vector and $\sigma_{x y}$ (vanishing when $\sigma_{x y}=0$ ).

We note that local current density patterns are usually thought to be obscured from view, and are difficult to detect directly via conventional probes. Indeed, optical probes typically access the plasmon's electric field and charge density configuration $[14,15]$. Nevertheless, the unusual plasmon dynamics and trajectories we discuss here manifest directly because of the current density texture that is hidden in the motion of electrons in the 2D metal plane yielding striking experimental signatures. This is akin to how (in electron transport) the inner (pseudo)spin degrees of freedom of an electron can alter its motion [9-11], or (in photonics) the spin-orbit interaction of light enables light polarizations to couple to its spatial degrees of freedom [22]. In the same way, a plasmon's current density configuration acts as an internal structure of the plasmon quasiparticle and enables unconventional dynamics.
We expect nontrivial plasmon textures to manifest in 2D metals with nonzero Hall conductivity, including conventional two-dimensional electron gas with an applied magnetic field, as well as anomalous Hall metals [23-25]. Further, the exposed 2D surface of these materials (e.g., those found in graphene heterostructures $[14,15,20,21])$ are a particularly ripe venue to observe the associated plasmon Hall shift and can be directly probed by scanning near-field scattering microscopy techniques $[14,15]$ recently developed to map out plasmon trajectories. These can provide a sensitive window into the internal structure of plasmon quasiparticles.

On a technological level, the nonreciprocal plasmon Hall shift and geometric phases provide new ways for routing plasmons. Indeed, achieving nonreciprocal plasmon propagation has been a subject of recent intense interest [23-31]. These schemes focus on utilizing asymmetric plasmon dispersion relations so that forward and backward propagating modes travel at different speeds or occur at different frequencies. Some examples include creating chiral oneway topological plasmonic edge modes [26,27], altering the forward and backward propagating plasmon dispersion by magnetic field [28], electrical current [29-31], Berry curvature of Bloch electrons [23,24], and pseudomagnetic field [25]. In contrast, the nonreciprocal plasmon Hall shifts we find appear even when the plasmon dispersion relation is fully symmetric [Fig. 1(b)]. Crucially, the nonreciprocity of plasmon Hall shifts arises from the unusual internal current density texture and not from the dispersion relation. This unusual effect of the internal structure of plasmon quasiparticles opens new means for manipulating plasmons in the extreme subwavelength regime.

\section{PLASMON CURRENT TEXTURE AND PSEUDOSPIN}

To analyze the geometric properties of plasmonic bands, we begin by examining its associated dynamical electric fields close to a 2D metal confined in the plane (at $z=0$ ). The dynamical evolution of electric fields $\mathcal{E}(\mathbf{r}, z, t)$ obeys Maxwell's equations so that

$\boldsymbol{\nabla} \times[\boldsymbol{\nabla} \times \mathcal{E}(\mathbf{r}, z, t)]=\frac{\omega^{2}}{c^{2}} \mathcal{E}(\mathbf{r}, z, t)-i \frac{4 \pi \omega}{c^{2}} \mathcal{J}(\mathbf{r}, z, t)$,

where $\mathbf{r}=(x, y)$ lie in the $2 \mathrm{D}$ plane, the current density $\mathcal{J}(\mathbf{r}, z, t)=\left(j_{x}, j_{y}, 0\right) \exp (i \omega t-i \mathbf{q} \cdot \mathbf{r}) \delta(z)$ is confined to the 2D metal, and $\mathbf{j}=\left(j_{x}, j_{y}\right)$ is the amplitude of the 2D current density. We have set the relative electric (magnetic) permittivity (permeability) to unity for simplicity.

Away from the 2D plane $(z \neq 0), \mathcal{J}(\mathbf{r}, z, t)$ vanishes, yielding electric fields that obey the familiar profile $\mathcal{E}(\mathbf{r}, z, t)=\mathbf{E} \exp \left(i \omega t-i \mathbf{q} \cdot \mathbf{r}-i k_{z} z\right)$ with $q_{x}^{2}+q_{y}^{2}+k_{z}^{2}=$ $\omega^{2} / c^{2}$, where $\mathbf{E}$ is a constant vector that denotes the polarization in the $2 \mathrm{D}$ plane and $\mathbf{q}=\left(q_{x}, q_{y}\right)$. Importantly, 
the free charge currents induced in the 2D plane enable tightly bound electromagnetic solutions close to the metallic plane [32]. In these plasmonic modes, $k_{z}^{2}<0$, so that the electric field amplitude decays exponentially away from $z=0$, so that $\mathcal{E}^{ \pm}(\mathbf{r}, z, t)=\mathbf{E}^{ \pm} \exp (\mp \beta z)$ $\exp (i \omega t-i \mathbf{q} \cdot \mathbf{r})$, where $\beta=\left(q^{2}-\omega^{2} / c^{2}\right)^{1 / 2}>0$, and $q^{2}=q_{x}^{2}+q_{y}^{2}$. The \pm superscripts denote the regions $z>0$ or $z<0$, respectively.

Substituting the form of the tightly bound fields into Eq. (1), we obtain electric fields in the 2D plane as

$$
\left(\begin{array}{c}
E_{x} \\
E_{y}
\end{array}\right)=\mathcal{F}\left(\begin{array}{c}
j_{x} \\
j_{y}
\end{array}\right), \quad \mathcal{F}=\frac{2 \pi i}{\omega \beta}\left(\begin{array}{cc}
\beta^{2}-q_{y}^{2} & q_{x} q_{y} \\
q_{x} q_{y} & \beta^{2}-q_{x}^{2}
\end{array}\right) .
$$

Plasmons (collective modes of the electron liquid) are obtained by the self-sustained solutions of Eq. (2) and the constitutive relations governing the transport of free carriers in the 2D plane, namely, its conductivity tensor: $\mathbf{j}=\boldsymbol{\sigma} \mathbf{E}$. Eliminating $\mathbf{E}$ in favor of the current density $\mathbf{j}$, we obtain 2D plasmons from solutions of

$$
\mathcal{M} \mathbf{j}=0, \quad \mathcal{M}=\mathcal{F}-\sigma^{-1},
$$

where the constitutive relation (encoded in $\boldsymbol{\sigma}$ ) determines both the dispersion and the configuration of current density in the 2D metal. As we display below, the current density configuration can wind as a function of plasmon propagation direction and wavelength leading to nontrivial geometrical effects.

We first consider plasmons in a conventional 2D metal with applied magnetic field $\mathbf{B}=B_{0} \hat{\mathbf{z}}$. Its in-plane conductivity is described by the Drude model:

$$
\sigma_{x x}=\frac{(1+i \omega \tau) \sigma_{0}}{(1+i \omega \tau)^{2}+\left(\omega_{c} \tau\right)^{2}}, \quad \sigma_{x y}=\frac{-\omega_{c} \tau \sigma_{0}}{(1+i \omega \tau)^{2}+\left(\omega_{c} \tau\right)^{2}},
$$

where $\sigma_{0}=D_{0} \tau$ is the static conductivity with $D_{0}=n e^{2} / m$ the Drude weight, $\tau$ is the scattering time, $m$ is the effective mass, and $\omega_{c}=e B_{0} / m$ is the cyclotron frequency.

Taking the nonretarded limit $(q \gg \omega / c)$ and analyzing the collisionless regime wherein $\tau \gg 1 / \omega$, we solve Eq. (3) using Eq. (4) to obtain the 2D bulk plasmon dispersion,

$$
\omega= \pm \omega_{q}, \quad \omega_{q}=\sqrt{a_{0} q+\omega_{c}^{2}}
$$

where $a_{0}=2 \pi D_{0}$. When $\omega_{c}=0, \omega_{q}$ yields the familiar 2D plasmon dispersion [see Fig. 1(c)]. $\omega_{c} \neq 0$ corresponds to the (gapped) bulk 2D magnetoplasmon spectrum [see Fig. 1(d)] [32]. Crucially, we find that the corresponding current density in the metal [eigenmodes of Eq. (3) in the $x-y$ basis] are

$$
\mathbf{u}^{ \pm}(\mathbf{q})=\left[\begin{array}{c}
j_{x}(\mathbf{q}) \\
j_{y}(\mathbf{q})
\end{array}\right]^{ \pm}=\frac{\mathcal{N}}{q}\left(\begin{array}{c}
\mp i q_{x}+\eta_{q} q_{y} \\
\mp i q_{y}-\eta_{q} q_{x}
\end{array}\right)
$$

Here, $\eta_{q}=\omega_{c} / \omega_{q} \in[-1,1]$ is a dimensionless parameter that captures the relative magnetic field strength and $\mathcal{N}=$ $\left(1+\eta_{q}^{2}\right)^{-1 / 2}$ is a normalization constant: $\left\langle\mathbf{u}^{ \pm}(\mathbf{q}) \mid \mathbf{u}^{ \pm}(\mathbf{q})\right\rangle=1$. We emphasize that $\eta_{q}$ represents the Hall coupling between the current $j_{x(y)}$ with its perpendicular electric field $E_{y(x)}$. We note, parenthetically, that the eigenmodes in each of the bands, $\mathbf{u}^{+}(\mathbf{q})$ and $\mathbf{u}^{-}(\mathbf{q})$, are locked to each other: $\left[\mathbf{u}^{+}(\mathbf{q})\right]^{*}=-\mathbf{u}^{-}(-\mathbf{q})$. This ensures that fields governed by Maxwell's equations are real valued (see Appendix A). Here and in the following, we focus on $\mathbf{u}(\mathbf{q})=\mathbf{u}^{+}(\mathbf{q})$ and drop the band index + for brevity.

Canting of current directions [see Eq. (6)] enables the plasmons to exhibit a chirality and acquire nontrivial phases as they propagate. To illustrate this, we analyze the pseudospin of the plasmon eigenmodes, $s_{i}(\mathbf{q})=$ $\left\langle\mathbf{u}(\mathbf{q})\left|\sigma_{i}\right| \mathbf{u}(\mathbf{q})\right\rangle$ :

$s_{1}=\frac{1-\eta_{q}^{2}}{1+\eta_{q}^{2}} \sin 2 \phi_{s}, \quad s_{2}=\frac{-2 \eta_{q}}{1+\eta_{q}^{2}}, \quad s_{3}=\frac{1-\eta_{q}^{2}}{1+\eta_{q}^{2}} \cos 2 \phi_{s}$,

where $\sigma_{i}$ are Pauli matrices $(i=1,2,3)$ and $\tan \phi_{s}=q_{y} / q_{x}$. As shown in Fig. 1(a), when $\omega_{c}=0$, conventional 2D metal plasmons possess pseudospin $\mathbf{s}$ components in the plane $\left(s_{2}=0, s_{1}, s_{3} \neq 0\right)$ and wind twice as $\phi_{s}$ is varied from 0 to $2 \pi$. In contrast, when $\omega_{c} \neq 0$, the pseudospin $\mathbf{s}$ cants out of the $\left(s_{1}, s_{3}\right)$ plane; see Figs. 1(b) and 1(d). While the pseudospin continues to wind twice as $\phi_{s}$ varies from $0 \rightarrow 2 \pi$, its $s_{2}$ component changes pitch, leading to a topologically nontrivial pseudospin texture. As we explain below, this hedgehoglike pseudospin texture enables plasmons to acquire nontrivial geometric phases as they propagate.

We note that in contrast to the nontrivial current density texture, electric field $\boldsymbol{\varepsilon}(\mathbf{q})$ directionality is insensitive to Hall conductivity and has orientation

$$
\boldsymbol{\varepsilon}(\mathbf{q})=\mathcal{F} \mathbf{u}(\mathbf{q})=\left(2 \pi \mathcal{N} / \omega_{q}\right) \mathbf{q},
$$

which continues to point along $\mathbf{q}$. As a result, the direction of $\boldsymbol{\varepsilon}(\mathbf{q})$ is the same for both plasmons at zero magnetic field and plasmons with a finite applied magnetic field; $\boldsymbol{\varepsilon}(\mathbf{q})$ plasmons are LE modes for all $\eta_{q}$.

\section{PLASMON GEOMETRIC PHASE AND BOUNDARY CONDITIONS}

We now illustrate the effect of the canted current density configuration in the dynamics of bulk plasmons. We consider a semi-infinite 2D metal (boundary at $x=0$ ) [see Figs. 2, 3(a), and 3(b)]; magnetic field is applied 


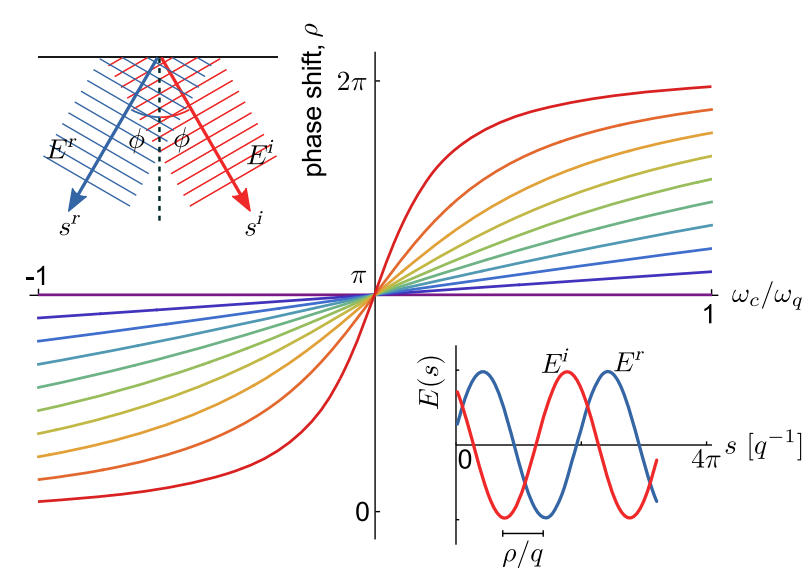

FIG. 2. Plasmon geometric phase. Phase shift between incident and reflected plane waves scattered off a boundary at different incident angles and relative magnetic field strength obtained from Eq. (11). While geometric phase shifts appear in both the current density and electric field, here we show the phase shift for (longitudinal) electric field along the directions $-\mathbf{q}^{i}\left(\mathbf{q}^{r}\right)$ away from the interface. Lines from purple (closest to the $x$ axis) to red (farthest away from the $x$ axis) correspond to incident angles $\phi_{n}=(\pi / 2)(n / 10)$ with $n$ integers in the range $n \in\{0, \ldots, 9\}$. Upper inset: Schematic for the incident and reflected plane wave. Lower inset: Electric field $E^{i}\left(E^{r}\right)$ of the incident (reflected) plane wave exhibits a nontrivial shift $\rho$ tunable by Hall conductivity (magnetic field).

along $\hat{\mathbf{z}}$. Plasmon waves that impinge on $x=0$ will get reflected elastically. In the main text, we focus on the regime of total reflection, see Appendix E for a more general discussion. To proceed, we construct the incident (reflected) plasmon wave fronts impinging on (moving away from) the boundary. The current density distribution is a linear superposition of eigenmodes $\mathbf{u}(\mathbf{q})$ :

$$
\mathbf{J}^{i, r}(\mathbf{r})=\sum_{n} g_{n}^{i, r} \mathbf{u}\left(\mathbf{q}_{n}^{i, r}\right) \exp \left(-i \mathbf{q}_{n}^{i, r} \cdot \mathbf{r}\right),
$$

where $g_{n}^{i, r}$ is a complex number that captures the current density amplitude and relative phases between components in the linear superposition.

First, we note that $\mathbf{q}_{n}^{i, r}$ for each component $n$ are related by translational symmetry along $y$ and energy conservation yielding $\left(q_{n, x}^{i}, q_{n, y}^{i}\right)=\left(-q_{n, x}^{r}, q_{n, y}^{r}\right)$. Importantly, $\mathbf{J}^{i, r}$ satisfies a current boundary condition along $x$ so that at the boundary the current vanishes:

$$
\hat{\mathbf{x}} \cdot\left[\mathbf{J}^{i}(x=0, y)+\mathbf{J}^{r}(x=0, y)\right]=0 .
$$

In order to satisfy Eq. (10), the complex superposition factors for incident and reflected waves obey the relation

$$
\frac{g_{n}^{r}}{g_{n}^{i}}=-\frac{\hat{\mathbf{x}} \cdot \mathbf{u}\left(\mathbf{q}_{n}^{i}\right)}{\hat{\mathbf{x}} \cdot \mathbf{u}\left(\mathbf{q}_{n}^{r}\right)}=-\exp \left(i \rho_{n}\right)
$$

where $\rho_{n}$ is a real number representing the phase shift between reflected and incident waves $\left(g_{n}^{r}\right.$ and $\left.g_{n}^{i}\right)$, because the norm of $-u_{x}\left(\mathbf{q}_{n}^{i}\right) / u_{x}\left(\mathbf{q}_{n}^{r}\right)$ is unity. We note that when $\eta_{q}=0$ (zero magnetic field), Eq. (11) yields the familiar trivial phase $\rho_{n}=\pi$. However, when $\eta_{q} \neq 0$ (finite magnetic field), $\rho_{n} \neq \pi$ and picking up a nontrivial phase. Importantly, since the phase shifts arise from the reflection coefficients, nontrivial $\rho_{n}$ manifest in both current density and electric field profiles. As a demonstration, we plot the phase shift $\rho$ in Fig. 2 for a plane wave scattering off the boundary. In the insets of Fig. 2, we have displayed the electric field profile obtained by applying Eq. (8) to Eq. (9). These exhibit nontrivial phases between incident $E^{i}$ and reflected $E^{r}$ waves when $\eta_{q} \neq 0$ (right-hand inset).

We emphasize that $\rho_{n}$ is directly related to the geometry of the canted pseudospin structure (Fig. 1). Writing $\rho_{n} \rightarrow \rho(\mathbf{q})$ as a continuous variable of incident wave vector $\mathbf{q}$, we find that the nontrivial phase $\rho(\mathbf{q})$ is intimately linked to a q-dependent geometric connection:

$$
\mathcal{A}_{\hat{\mathbf{n}}}(\mathbf{q})=\left\langle u_{\hat{\mathbf{n}}}(\mathbf{q})\left|i \nabla_{\mathbf{q}}\right| u_{\hat{\mathbf{n}}}(\mathbf{q})\right\rangle,
$$

via $\quad \nabla_{\mathbf{q}} \rho(\mathbf{q})=\mathcal{A}_{\hat{\mathbf{n}}}\left(\mathbf{q}_{0}^{r}\right)-\mathcal{A}_{\hat{\mathbf{n}}}\left(\mathbf{q}_{0}^{i}\right), \quad$ where $\quad\left|u_{\hat{\mathbf{n}}}(\mathbf{q})\right\rangle=$ $|u(\mathbf{q}) \cdot \hat{\mathbf{n}}\rangle\langle\hat{\mathbf{n}} \cdot u(\mathbf{q}) \mid u(\mathbf{q}) \cdot \hat{\mathbf{n}}\rangle^{-1 / 2}$, and $\hat{\mathbf{n}}$ is a unit vector denoting the direction of the interface [for $\rho(\mathbf{q})$ defined by the boundary; in Eq. (11), $\hat{\mathbf{n}}$ is $\hat{x}]$. We note that $\mathbf{u}(\mathbf{q})$ are classical eigenmodes and do not possess gauge freedom. As a result, the corresponding geometric connection $\mathcal{A}_{\hat{\mathbf{n}}}(\mathbf{q})$ is fixed in each system (characterized by its conductivity tensor, $\boldsymbol{\sigma})$, and is real [33].

\section{PLASMON HALL SHIFT}

As we now show, the nontrivial phases acquired above can build up to dramatic effect for wave packets (superposition of plane waves). In particular, wave packets reflected off a boundary experience a real-space shift [see Fig. 3(b)] with the reflected wave packet center translated along the boundary when a magnetic field is applied (relative to the zero magnetic field case): the plasmon Hall shift.

To clearly illustrate the plasmon Hall shift, we analyze wave packets of electric field strength $\mathbf{E}(\mathbf{r}$ ) (see Appendix $\mathrm{F}$ for other types of wave packets). Since the intensity distribution $|\mathbf{E}(\mathbf{r})|^{2}$ can be directly measured by scanning near-field optical probes $[14,15]$, we focus on $\left|\mathbf{E}^{i, r}(\mathbf{r})\right|^{2}$ peak position to track the location of the plasmon wave packet in real space. The electric field strength $\mathbf{E}(\mathbf{r})$ of the plasmon wave packet can be directly obtained from Eq. (9) via Eq. (8). In constructing the wave packet we take a continuous superposition of eigenwaves so that the incident complex superposition factors $g_{n}^{i} \rightarrow f(\mathbf{q})=$ $|f(\mathbf{q})| \exp [i \delta(\mathbf{q})]$, where $|f(\mathbf{q})|$ is real with a narrow and single center at $\mathbf{q}_{0}=\left(q_{0} \cos \phi_{0}, q_{0} \sin \phi_{0}\right)$ with $\phi_{0}$ the incident angle and $\delta(\mathbf{q})$ is the phase distribution profile. We obtain 


$$
\mathbf{E}^{i, r}(\mathbf{r})=\int d \mathbf{q} f(\mathbf{q}) p^{i, r}(\mathbf{q}) \boldsymbol{\varepsilon}^{i, r}(\mathbf{q}) e^{\mp i q_{x} x-i q_{y} y}
$$

where $\boldsymbol{\varepsilon}^{i}(\mathbf{q})=\boldsymbol{\varepsilon}\left(q_{x}, q_{y}\right)$ corresponds to the incident wave, $\boldsymbol{\varepsilon}^{r}(\mathbf{q})=\boldsymbol{\varepsilon}\left(-q_{x}, q_{y}\right)$ corresponds to the reflected wave, and $p^{i}(\mathbf{q})$ and $p^{r}(\mathbf{q})$ are the incident and reflection coefficients for each $\mathbf{q}$ component, respectively. Setting $p^{i}(\mathbf{q})=1$ without loss of generality, we can write $g_{n}^{r} / g_{n}^{i} \rightarrow$ $p^{r}(\mathbf{q})=-\exp [i \rho(\mathbf{q})]$. As we see, accumulation of this phase difference $\rho(\mathbf{q})$ enables the inner geometric structure of the plasmon [see Eq. (12)] to affect its propagation.

Using Eq. (13), we track the center of the electric field intensity $\left[\operatorname{Re} \mathbf{E}^{i, r}(\mathbf{r})\right]^{2}$. We determine the center of the narrowly distributed wave packet (beam) using the standard method of stationary phases (see Appendixes B and C), obtaining the trajectories for the center of the incident $y_{c}^{i}(x)$ and reflected $y_{c}^{r}(x)$ beams as

$y_{c}^{i}(x)=-\kappa_{0} x+y_{0}, \quad y_{c}^{r}(x)=\kappa_{0} x+y_{0}+\Delta y_{H}$,

where $\kappa_{0}=-\tan \phi_{0}$. Here, $y_{0}=\left[d \delta(\mathbf{q}) / d q_{y}\right]_{\mathbf{q}=\mathbf{q}_{0}}$ denotes a reference point (e.g., absolute position of the wave packet along $y$ ) fixed by the relative phase factors in the complex superposition of the incident wave packet.

Importantly, the incident and reflected trajectories, $y_{c}^{i, r}$, are displaced at $x=0$ by $\Delta y_{H}$. This Hall shift arises from the nontrivial geometric phases [Eq. (11)] sustained by each q component of the wave packet as it is reflected off the boundary. Since phases are slightly mismatched for different $\mathbf{q}$, their phase gradient produces

$$
\begin{aligned}
\Delta y_{H} & =\left[d \rho(\mathbf{q}+\mathbf{k}) / d k_{y}\right]_{\mathbf{q}=\mathbf{q}_{0}, \mathbf{k}=0} \\
& =\kappa_{0}\left[\mathcal{A}_{\hat{x}}\left(\mathbf{q}_{0}^{r}\right)-\mathcal{A}_{\hat{x}}\left(\mathbf{q}_{0}^{i}\right)\right]_{x}+\left[\mathcal{A}_{\hat{x}}\left(\mathbf{q}_{0}^{r}\right)-\mathcal{A}_{\hat{x}}\left(\mathbf{q}_{0}^{i}\right)\right]_{y},
\end{aligned}
$$

where we have used Eqs. (11) and (12). Equation (15) shows that the real-space shift is directly related to the geometric properties of the plasmon band, allowing direct access into its internal structure via real-space mapping.

Using the form of the current density $u_{x}$ in Eqs. (6) and (12), we find the Hall shift for 2D magnetoplasmons as

$$
\Delta y_{H}^{\mathrm{mp}}=\frac{1}{q_{0}} \frac{2 \eta_{0} \sec \phi_{0}}{\cos ^{2} \phi_{0}+\eta_{0}^{2} \sin ^{2} \phi_{0}},
$$

where $\eta_{0}=\omega_{c} / \omega_{q_{0}}$. An explicit calculation of $\Delta y_{H}^{\mathrm{mp}}$ using Gaussian wave packets reproduces Eq. (16); see Appendix D. We note that $\Delta y_{H}^{\mathrm{mp}}$ [Fig. 3(c)] is controlled by $\eta_{0}$, and vanishes in the presence of time reversal symmetry (e.g., at $\omega_{c}=0$ ). Further, it exhibits a sharp $\phi_{0}$ dependence with $\Delta y_{H}^{\mathrm{mp}}$ allowing the effect to be further tuned.

Curiously, although plasmons are charge neutral excitations, they can still experience a Hall shift similar to that

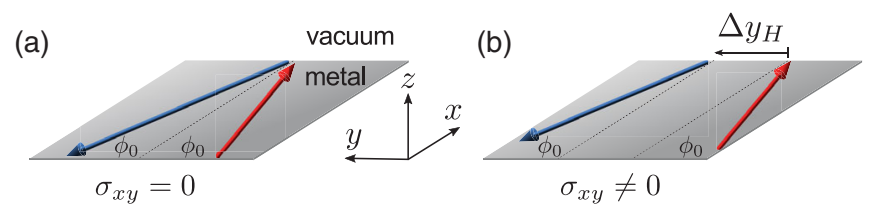

(c)
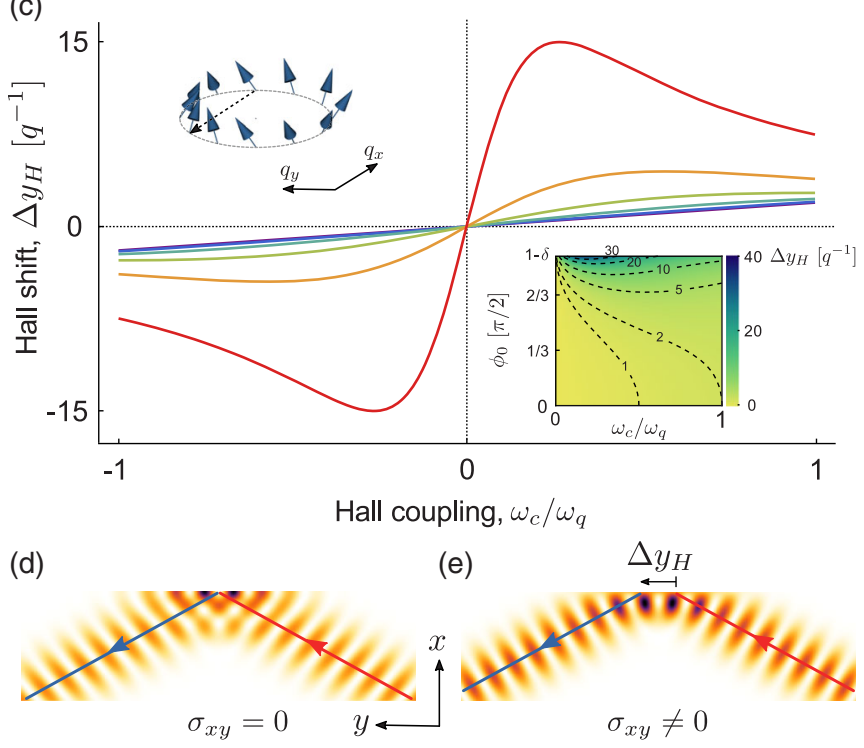

FIG. 3. Plasmon Hall shift. (a),(b) Schematic of plasmon Hall shift between the incident and reflected wave packets with incident angle $\phi_{0}$. The Hall shift $\Delta y_{H}$ is nonzero when $\sigma_{x y} \neq 0$. (c) Plasmon Hall shift for $2 \mathrm{D}$ magnetoplasmons (in units of inverse wave vector $q^{-1}$ ) from Eq. (16) at various incident angles $\phi_{0}$ can be tuned by the Hall coupling $\eta=\omega_{c} / \omega_{q}$. Lines from purple (closest to the line $\Delta y_{H}=0$ ) to red (farthest away from the line $\left.\Delta y_{H}=0\right)$ correspond to incident angles $\phi_{n}=$ $(\pi / 2)(n / 6)$ with $n$ integers in the range $n \in\{0, \ldots, 5\}$. The upper inset displays the pseuodospinor orientation on a bulk plasmon isofrequency contour highlighting the different orientations between incident and reflected waves. Lower inset: Contour plot of Hall shift as a function of Hall coupling and $\phi_{0}$. We have used $\delta=\pi / 20$. (d),(e) Numerically computed electric field intensity $\left[\operatorname{Re} \mathbf{E}^{i}(\mathbf{r})+\operatorname{Re}^{r}(\mathbf{r})\right]^{2}$ from Eq. (13), without and with magnetic field (at $\eta_{q}=1$ limit), for an incident wave with a Gaussian distribution and fixed energy. The width of the wave is $\pi / q$ and the incident angle is $\pi / 3$, with a Hall shift $4 / q$. Red and blue lines are beam trajectories obtained from Eq. (14).

of charged particles. Charged particles experience a transverse flow that depends on the sign of the charge, applied electric field, and magnetic field direction. The direction of the plasmon Hall shift above is determined by the Hall coupling (set by the Hall conductivity) and the direction of the wave vector perpendicular to the boundary $q_{x}$, giving $\operatorname{sgn}\left(\Delta y_{H}\right)=\operatorname{sgn}\left(\eta_{q} q_{x}\right)$. As a result, $\Delta y_{H}$ is even in $\phi_{0}$, yielding the same direction of shift for both positive and negative $\phi_{0}$ plasmon waves incident on a single boundary. The nonreciprocal shift of bulk plasmon wave packets provides a novel way of filtering plasmons that propagate in opposite directions in $q_{y}$ (along the boundary) but with 
the same sign in $q_{x}$ (perpendicular to the boundary) see discussion below.

\section{ANOMALOUS PLASMON HALL SHIFT}

While we focused on conventional 2D metals at finite magnetic field above, a nontrivial plasmon band geometry can also be achieved at zero field. For example, plasmon excitations can be supported in anomalous Hall metals [23], electrons in strained graphene or in proximity to a Skyrmion lattice [25], as well as out-of-equilibrium systems $[23,24]$, all of which possess a Hall conductivity in the absence of an applied magnetic field.

To illustrate the anomalous plasmon Hall shift, we concentrate on the first example: anomalous Hall metals. We take a simple phenomenological model for the conductivity tensor with longitudinal component $\sigma_{x x}=$ $\sigma_{0} /(1+i \omega \tau)$ in the usual Drude form and Hall conductivity $\sigma_{\mathrm{AH}}$ (valid for small frequencies and wave vectors). Following the same procedure as in Eqs. (1)-(3) above, we obtain a gapless bulk plasmon frequency as $\omega_{q}^{A}=\sqrt{a_{0} q}$, where $a_{0}=2 \pi D_{0}$. Furthermore, just as conventional Hall conductivity (from a Lorentz force) skews the current density orientation (see Fig. 1), anomalous Hall conductivity also yields a canted current density orientation. The current density orientation for anomalous Hall plasmon eigenmodes maps precisely to Eq. (6), but with $\eta_{q}=\eta_{q}^{A}=\omega_{q}^{A} \sigma_{\mathrm{AH}} / D_{0}$.

As a result of the nontrivial bulk plasmon current density texture, bulk plasmons in anomalous Hall metals also experience a plasmon Hall shift. Indeed, applying the eigenmodes obtained above, and considering the same geometry as in Fig. 3, we obtain the Hall shift

$$
\Delta y_{H}^{A}=\frac{1}{q_{0}} \frac{2 \eta_{0}^{A} \sec \phi_{0}}{\cos ^{2} \phi_{0}+\left(\eta_{0}^{A}\right)^{2} \sin ^{2} \phi_{0}},
$$

which is controlled by incident wave vector $\mathbf{q}_{0}=$ $\left(q_{0} \cos \phi_{0}, q_{0} \sin \phi_{0}\right)$, and dimensionless Hall coupling $\eta_{0}^{A}=\omega_{q_{0}}^{A} \sigma_{\mathrm{AH}} / D_{0}$. In contrast to the case of magnetoplasmons above, we note the Hall coupling $\eta_{0}^{A}$ can be larger than unity. As a result, anomalous Hall plasmons may enable access to larger Hall shifts.

\section{DISCUSSION}

The plasmon Hall shifts we discuss here can be probed by a variety of experimental techniques. For example, a focused plasmon wave packet can be launched at a reflecting boundary (e.g., via designer nanoanttenas [34] fixed on the sample). The subsequently reflected plasmon wave packet can be tracked by outcoupling light using scanning near-field tip and microscope [14,15], or detected electrically [35] (plasmons incident on a $p-n$ junction can excite hot carriers yielding a theromoelectric current that can be measured via conventional dc electrical transport). Since the Hall shift is tunable by both incident angle and wave vector $\mathbf{q}$, as well as an applied magnetic field, the reflected plasmon trajectory can enable an optical mapping of the inner structure of bulk plasmon bands, namely, its current density orientation or texture. This is similar to the role spin-resolved photoemission spectroscopy plays in mapping the spinor structure of topologically nontrivial electronic band structures.

The nonreciprocity of the plasmon Hall shift can enable an unusual type of (nonreciprocal) plasmon wave filter, sketched in Fig. 4. In this setup, focused plasmon beams are launched and are incident on a reflecting boundary (red arrows). Upon reflection, the reflected beams (blue arrows) are translated by $\Delta y_{H}$ that is fixed by the direction of the magnetic field. For example, in Fig. 4(a), beams incident from the left (forward propagating) are boosted in the forward direction (top panel), whereas plasmon beams incident from the right (reverse propagating) are translated backward (bottom panel). By placing a plasmon-absorbing region (gray shaded area) close to the boundary, reverse propagating beams in Fig. 4(a) can be filtered out whereas forward propagating beams are unhindered. The plasmonabsorbing regions can be created by various means, including selectively adding disorder to the sample only within that region, thus damping the plasmons that pass through. The reflected beam intensity can be collected, e.g., by a $p-n$ junction thermoelectric detector fabricated close by [35]. Similarly, by changing the sign of the magnetic field in Fig. 4(b), forward propagating beams are filtered, whereas reverse propagating beams pass
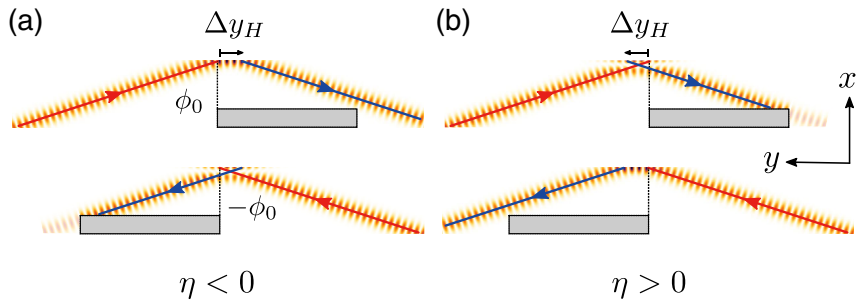

FIG. 4. Nonreciprocal plasmon filter. (a), (b) Plasmon Hall shift displacement at a fixed $\eta=\omega_{c} / \omega_{q}$ are identical for beams with incident angle $\phi_{0}$ and $-\phi_{0}$. Gray regions are areas inside which plasmons damp quickly; see text. For plasmon beams incident from the left-hand (right-hand) side, the reflected beam can bypass the damping region when $\eta$ is negative (positive); these reflected beams can be collected at an all-electrical plasmon detector stationed close by ( $p-n$ junction thermoelectric detector [35]). In contrast, plasmon beams incident from the right-hand (left-hand) side pass through the damping region and are damped when $\eta$ is negative (positive). Red (incident) and blue (reflected) lines are beam trajectories obtained from Eq. (14). Parameters used are as follows: the width of the wave is $\pi / q$, the same as Fig. $3(\mathrm{~d}), \phi_{0}=2 \pi / 5$, and $\omega_{c} / \omega_{q}= \pm \cot \phi_{0}$ is taken to maximize the plasmon Hall shift. 
through unhindered. This is made possible by the nonreciprocity of the plasmon Hall shift (fixed by magnetic field), and opens new ways for plasmon path engineering in plasmonic devices.

We note that the plasmon Hall shift while reminiscent of the Hall effect of light $[5,6]$ is distinct from it in both its phenomenology and microscopic origin. First, the plasmons in 2D metals we consider are deep in the nonretarded regime exhibiting a dramatically compressed wavelength than that of light. This renders the plasmon Hall shifts discussed above far below the diffraction limit; wave packet shifts of light are typically on the order of the free space wavelength $[5,6]$. Second, geometrical phases in optics and photonics (in the retarded limit) arise from a texture in the electric polarization of light [22]. In contrast, deep in the nonretarded limit, plasmons are purely composed of LE modes (electric field aligned with q) which possess identical orientations for $\sigma_{x y}=0$ metals and $\sigma_{x y} \neq 0$ metals. Nontrivial plasmon geometric phases instead arise from a texture of the current density characterizing the collective motion of electrons in the 2D metal plane; this current density texture is unique to plasmon modes in metals and can be viewed as a "hidden" internal degree of freedom hosted within the 2D metallic plane clearly delineating the behavior of plasmons in $\sigma_{x y}=0$ metals and $\sigma_{x y} \neq 0$ metals. Lastly, we note that geometrical phases acquired by plasmons enable their motion to be controlled by conductivity and a homogeneous applied magnetic field. This provides an on-demand means of tuning the dynamics of plasmon wave packets.

We expect that plasmon Hall shifts $\Delta y_{H}$ can be readily realized in high-quality two-dimensional metallic systems for, e.g., those found in existing graphene/hexagonal-boron nitride $(\mathrm{G} / \mathrm{hBN})$ heterostructures $[14,15,20]$ under reasonable magnetic fields. To see this, we first note that in the presence of plasmon loss (e.g., through disorder scattering, phonon scattering) the amplitude of the plasmon oscillations decays. This is characterized by a quality factor $Q$ that determines the number of cycles a plasmon oscillates through before it decays. In high-quality graphene samples, large $Q \sim 10-100$ have been reported [14,15,20,21]. As a result, we anticipate that plasmon Hall shifts as large as $Q \lambda$ can be detected in these systems. Here, $\lambda=2 \pi /|\mathbf{q}|$ is the plasmon wavelength. Next, we note that the largest plasmon Hall shifts occur when the incident angle $\phi_{0}>\pi / 4$ [see Eq. (16) and Fig. 3]. For example, for incident angle $\phi_{0}=5 \pi / 12$, the peak Hall shift occurs at $\Delta y_{H}=2.46 \lambda$. For a graphene sample, and choosing $\hbar \omega_{q}=50 \mathrm{meV}$ plasmon excitation for a typical carrier density $n=10^{12} \mathrm{~cm}^{-2}$, the peak Hall shift occurs at magnetic field $B=2.37 \mathrm{~T}$ (see Appendix $\mathrm{H}$ ). For larger incident angles, the required magnetic fields to achieve peak Hall shift values can be even smaller.

In summary, the internal current density configuration of plasmons in a metal can be transformed by transverse
Hall conductivity and, as a result, enable a new set of plasmon kinematics. In particular, we find that $\sigma_{x y} \neq 0$ plasmon wave packets reflected off a boundary can acquire a nonreciprocal Hall shift parallel to the boundary. While we focus on the plasmon Hall shift here, we expect that nontrivial plasmon band geometry can also enable a wealth of unconventional dynamics not available when $\sigma_{x y}=0$. For example, geometric phases can be accumulated by plasmon wave packets as they propagate through inhomogeneous media, and may lead to anomalous plasmon wave packet dynamics (e.g., anomalous velocity) in much the same way as for wave packets of electrons $[9,10,36]$ and photons $[5,6,13]$; it can also lead to a topological structure of plasmon bands [26]. Nontrivial band geometry provides a new toolbox in which to control the dynamics of plasmons, as well as a window through which to view the internal structure governing the nanophotonic properties of materials.

\section{ACKNOWLEDGMENTS}

We thank Eddwi Hesky Hasdeo and Yidong Chong for useful discussions. We are grateful to Alessandro Principi for a critical reading. This work was supported by the Singapore National Research Foundation (NRF) under NRF fellowship Grant No. NRF-NRFF2016-05. L.-k. S. and J.C.W.S. designed the research, performed the research, and wrote the manuscript.

\section{APPENDIX A: RELATION BETWEEN EIGENMODES $\mathbf{u}^{+}(\mathbf{q})$ AND $\mathbf{u}^{-}(\mathbf{q})$}

The eigenmodes $\mathbf{u}^{+}(\mathbf{q})$ and $\mathbf{u}^{-}(\mathbf{q})$ in the main text are complex conjugate to each other: $\left[\mathbf{u}^{+}(\mathbf{q})\right]^{*}=-\mathbf{u}^{-}(-\mathbf{q})$. This ensures that fields governed by Maxwell's equations are real valued. To see this, consider a real-valued current field:

$\mathbf{J}(\mathbf{r}, t)=\frac{1}{2}\left[\mathbf{u}^{+}(\mathbf{q}) e^{i \omega_{q} t-i \mathbf{q} \cdot \mathbf{r}}-\mathbf{u}^{-}(-\mathbf{q}) e^{i\left(-\omega_{q}\right) t-i(-\mathbf{q}) \cdot \mathbf{r}}\right]$.

The corresponding electric field can be directly obtained from Eq. (A1) by applying $\mathcal{F}$, which is an operator that depends on $\omega$ :

$$
\begin{aligned}
\mathbf{E}(\mathbf{r}, t) & =\mathcal{F} \mathbf{J}(\mathbf{r}, t) \\
& =\frac{\mathcal{F}}{2}\left[\mathbf{u}^{+}(\mathbf{q}) e^{i \omega_{q} t-i \mathbf{q} \cdot \mathbf{r}}-\mathbf{u}^{-}(-\mathbf{q}) e^{i\left(-\omega_{q}\right) t-i(-\mathbf{q}) \cdot \mathbf{r}}\right] \\
& =\frac{1}{2}\left[\boldsymbol{\varepsilon}(\mathbf{q}) e^{i \omega_{q} t-i \mathbf{q} \cdot \mathbf{r}}-\boldsymbol{\varepsilon}(-\mathbf{q}) e^{i\left(-\omega_{q}\right) t-i(-\mathbf{q}) \cdot \mathbf{r}}\right] \\
& =\boldsymbol{\varepsilon}(\mathbf{q}) \cos \left(\omega_{q} t-\mathbf{q} \cdot \mathbf{r}\right),
\end{aligned}
$$

where we note that $\boldsymbol{\varepsilon}(\mathbf{q})=\mathcal{F} \mathbf{u}^{ \pm}(\mathbf{q})$. This latter relationship and the fact that $\varepsilon(\mathbf{q})$ is the same for both 
+ and - bands can be directly verified [using Eqs. (2), (6), and (8) of the main text]:

$$
\begin{aligned}
\mathcal{F} \mathbf{u}^{ \pm}(\mathbf{q}) & =\frac{2 \pi i}{ \pm \omega_{q} q}\left(\begin{array}{cc}
q_{x}^{2} & q_{x} q_{y} \\
q_{x} q_{y} & q_{y}^{2}
\end{array}\right) \frac{\mathcal{N}}{q}\left(\begin{array}{l}
\mp i q_{x}+\eta_{q} q_{y} \\
\mp i q_{y}-\eta_{q} q_{x}
\end{array}\right) \\
& =\frac{2 \pi \mathcal{N}}{ \pm \omega_{q}} \frac{i}{q^{2}}\left(\begin{array}{c}
\mp i q_{x}\left(q_{x}^{2}+q_{y}^{2}\right) \\
\mp i q_{y}\left(q_{x}^{2}+q_{y}^{2}\right)
\end{array}\right) \\
& =\frac{2 \pi \mathcal{N}}{\omega_{q}}\left(\begin{array}{c}
q_{x} \\
q_{y}
\end{array}\right)=\boldsymbol{\varepsilon}(\mathbf{q}) .
\end{aligned}
$$

As a result, $\mathbf{E}(\mathbf{r}, t)$ in Eq. (A2) is also a real-valued field; this arises because $\left[\mathbf{u}^{+}(\mathbf{q})\right]^{*}=-\mathbf{u}^{-}(-\mathbf{q})$. Here we note that $\varepsilon(\mathbf{q})$ is purely real, as shown in Eq. (A3).

It is useful to note that the requirement of real-valued fields guarantees the existence of both $\mathbf{u}^{+}(\mathbf{q})$ and $\mathbf{u}^{-}(\mathbf{q})$ bands. Further, as is displayed in Eq. (A3), $\boldsymbol{\varepsilon}(\mathbf{q})$ is a pure longitudinal electric mode (LE), where the (in-plane) electric field points in the same direction as the propagation direction.

\section{APPENDIX B: ENVELOPE FOR ELECTRIC FIELD INTENSITY}

In the main text, we have the incident and reflected wave packets for electric fields:

$$
\mathbf{E}^{i, r}(\mathbf{r})=\int d \mathbf{q} f(\mathbf{q}) p^{i, r}(\mathbf{q}) \boldsymbol{\varepsilon}^{i, r}(\mathbf{q}) e^{\mp i q_{x} x-i q_{y} y},
$$

where $f(\mathbf{q})=|f(\mathbf{q})| \exp [i \delta(\mathbf{q})]$, with $|f(\mathbf{q})|$ and $\delta(\mathbf{q})$ the amplitude and phase distribution profiles in $\mathbf{q}$ space, respectively. Here, $p^{i}(\mathbf{q})$ and $p^{r}(\mathbf{q})$ are the incident and (complex) reflection coefficients for each $\mathbf{q}$ component, respectively. The intensities of the incident and reflected waves in real space are squares of their real parts. Expressing $p^{r}(\mathbf{q})=-\exp [i \rho(\mathbf{q})]$ as an exponential phase factor [we set $p^{i}(\mathbf{q})=1$ without loss of generality], we have the intensity

$$
\begin{aligned}
{\left[\operatorname{Re} \mathbf{E}^{i, r}(\mathbf{r})\right]^{2}=} & \operatorname{Re} \int d \mathbf{q} d \mathbf{k} f_{E}^{i, r}(\mathbf{q}, \mathbf{k}) e^{i \theta^{i, r}(\mathbf{q}, \mathbf{k}, \mathbf{r})} \\
& \times \frac{1+e^{i \theta_{+}^{i, r}(\mathbf{q}, \mathbf{r})}}{2}
\end{aligned}
$$

where $f_{E}^{i, r}(\mathbf{q}, \mathbf{k})=|f(\mathbf{q})||f(\mathbf{q}+\mathbf{k})| \boldsymbol{\varepsilon}^{i, r}(\mathbf{q}) \boldsymbol{\varepsilon}^{i, r}(\mathbf{q}+\mathbf{k})$ is a real function of $(\mathbf{q}, \mathbf{k})$, and

$$
\begin{aligned}
\theta^{i} & =-k_{x} x-k_{y} y+\Delta \delta(\mathbf{q}, \mathbf{k}), \\
\theta^{r} & =+k_{x} x-k_{y} y+\Delta \delta(\mathbf{q}, \mathbf{k})+\Delta \rho(\mathbf{q}, \mathbf{k}), \\
\theta_{+}^{i} & =-2 q_{x} x-2 q_{y} y+2 \delta(\mathbf{q}), \\
\theta_{+}^{r} & =+2 q_{x} x-2 q_{y} y+2 \delta(\mathbf{q})+2 \rho(\mathbf{q}) .
\end{aligned}
$$

Here, $\quad \Delta \delta(\mathbf{q}, \mathbf{k})=\delta(\mathbf{q}+\mathbf{k})-\delta(\mathbf{q}) \quad$ and $\quad \Delta \rho(\mathbf{q}, \mathbf{k})=$ $\rho(\mathbf{q}+\mathbf{k})-\rho(\mathbf{q})$.

Because $f_{E}^{i, r}(\mathbf{q}, \mathbf{k})$ is peaked around $\left(\mathbf{q}=\mathbf{q}_{0}, \mathbf{k}=0\right)$, $\exp \left[i \theta_{+}^{i . r}(\mathbf{q})\right] \sim \exp \left(\mp 2 i q_{x} x-2 i q_{y} y\right)$ is highly oscillatory. This contrasts with the slowly varying envelope function $\exp \left[i \theta^{i, r}(\mathbf{q}, \mathbf{k}, \mathbf{r})\right] \sim \exp \left(\mp i k_{x} x-i k_{y} y\right)$. As a result, to describe the wave packet centers, we focus on the envelope function to track the slowly varying electric field intensities as shown in Eq. (C1).

\section{APPENDIX C: WAVE PACKET CENTER AND STATIONARY PHASES}

In this appendix, we detail the standard method of stationary phases used to determine the wave packet center and its trajectory. Using Eq. (B2) [see also Eq. (13) of the main text], we focus on the slowly varying envelope for electric field intensity $\left[\operatorname{Re} \mathbf{E}^{i, r}(\mathbf{r})\right]^{2}$ (see the discussion in Appendix B) that can be expressed as

$$
F^{i, r}(\mathbf{r})=\int d \mathbf{q} d \mathbf{k} f_{E}^{i, r}(\mathbf{q}, \mathbf{k}) \exp \left[i \theta^{i, r}(\mathbf{q}, \mathbf{k}, \mathbf{r})\right],
$$

where $f_{E}^{i, r}(\mathbf{q}, \mathbf{k})=|f(\mathbf{q}) \| f(\mathbf{q}+\mathbf{k})| \boldsymbol{\varepsilon}^{i, r}(\mathbf{q}) \cdot \boldsymbol{\varepsilon}^{i, r}(\mathbf{q}+\mathbf{k})$ is a real function of $(\mathbf{q}, \mathbf{k})$, and

$$
\begin{aligned}
& \theta^{i}=\Delta \delta(\mathbf{q}, \mathbf{k})-k_{x} x-k_{y} y, \\
& \theta^{r}=\Delta \delta(\mathbf{q}, \mathbf{k})+k_{x} x-k_{y} y+\rho(\mathbf{q}+\mathbf{k})-\rho(\mathbf{q}),
\end{aligned}
$$

where $\Delta \delta(\mathbf{q}, \mathbf{k})=\delta(\mathbf{q}+\mathbf{k})-\delta(\mathbf{q})$. In what follows, we analyze Eq. (C1) for $|f(\mathbf{q})|$ that is narrowly distributed about $\mathbf{q}_{0}$.

For clarity, we first concentrate on the case of a single frequency [11,37-41] valid for plasmon beams with a narrow frequency width. In this case, $\boldsymbol{\varepsilon}^{i, r}(\mathbf{q}) \cdot \boldsymbol{\varepsilon}^{i, r}(\mathbf{q}+\mathbf{k})=$ $\left(2 \pi \mathcal{N} q_{0} / \omega_{0}\right)^{2} \cos \varphi$, with $\varphi$ the angle between $\mathbf{q}$ and $\mathbf{q}+\mathbf{k}$. As a result, $f_{E}^{i}(\mathbf{q}, \mathbf{k})=f_{E}^{r}(\mathbf{q}, \mathbf{k})$ are sharply peaked about $\left(\mathbf{q}=\mathbf{q}_{0}, \mathbf{k}=0\right)$. The center or peak of $F^{i, r}(x, y)$ in the $y$ direction at a given $x$, denoted $y_{c}^{i, r}(x)$, can be obtained directly via the method of stationary phases:

$$
\left[d \theta^{i, r}(\mathbf{q}, \mathbf{k}, x, y) / d k_{y}\right]_{\mathbf{q}=\mathbf{q}_{0}, \mathbf{k}=0}=0,
$$

so that for $y=y_{c}^{i, r}$, the phases $\theta^{i, r}$ in Eq. (C1) add constructively, yielding a maximal $F^{i, r}(x, y)$. By directly taking derivatives, Eq. (C3) yields the incident and reflected wave packet center as described in Eq. (14) of the main text, with

$$
\kappa_{0}=\left[d k_{x} / d k_{y}\right]_{\mathbf{q}=\mathbf{q}_{0}, \mathbf{k}=0}=-q_{0, y} / q_{0, x}=-\tan \phi,
$$

where $k_{x}=\sqrt{q_{0}^{2}-\left(q_{y}+k_{y}\right)^{2}}-q_{x}$, and $\phi$ denotes the incident angle. For an explicit calculation of $y_{c}^{i, r}(x)$ using 
Gaussian wave packets, see Appendix D. This reproduces all our results.

For a discussion of wave packets possessing a distribution of frequencies, see Appendix G. For narrow frequency distributions, these yield the same results as discussed in the main text.

\section{APPENDIX D: PLASMON HALL SHIFT USING GAUSSIAN WAVE PACKETS}

The formulation discussed in the main text reveals a plasmon Hall shift that is insensitive to the details of the profile $f(\mathbf{q})$. For concreteness, here we calculate the plasmon Hall shift for a specific wave packet profile: Gaussian wave packets. To proceed we construct an incident wave packet composed of $\mathbf{u}(\mathbf{q})$ modes, with a fixed frequency $\omega_{q}=\omega_{0}$ and a distribution of incident angles $\phi$ which satisfy a Gaussian distribution $f(\phi)=$ $\exp \left[-\left(\phi-\phi_{0}\right)^{2} / 2 \ell^{2}\right]$ of width $\ell$. Here we set $\delta(\phi)=0$ without loss of generality, since it corresponds to a reference point $y_{0}=0$ [see Eq. (14) of the main text]. From Eq. (C1), the envelope function for incident or reflected electric field intensity is

$$
\begin{aligned}
F^{i, r}(y)= & \int d \phi d \varphi f(\phi) f(\phi+\varphi) p^{i, r}(\phi)^{*} p^{i, r}(\phi+\varphi) \\
& \times \boldsymbol{\varepsilon}^{i, r}(\phi) \boldsymbol{\varepsilon}^{i, r}(\phi+\varphi) \exp \left(-i k_{y} y\right),
\end{aligned}
$$

where $\boldsymbol{\varepsilon}^{i, r}(\phi) \boldsymbol{\varepsilon}^{i, r}(\phi+\varphi)=\varepsilon_{0}^{2} \cos \varphi$, with $\varepsilon_{0}=2 \pi \mathcal{N}_{0} q_{0} / \omega_{0}$, $k_{y}=q_{0}[\sin (\phi+\varphi)-\sin \phi]$. Setting the incident coefficient $p^{i}(\phi)=1$, the reflection coefficient is

$$
p^{r}(\phi)=\frac{i \cos \phi-\eta_{0} \sin \phi}{i \cos \phi+\eta_{0} \sin \phi},
$$

with $\eta_{0}=\omega_{c} / \omega_{0}$. In Eq. (D1), we have assumed a narrow beam profile with $\ell \ll 1$. This allows us to extend the integral limits of $\phi$ and $\varphi$ from $[-\pi, \pi]$ to $(-\infty, \infty)$. Further, we have set $x=0$ since we are interested in the plasmon Hall shift (shift between $y_{c}^{i}$ and $y_{c}^{r}$ position) along the boundary $x=0$.

Because $(\phi, \varphi)$ contributes mostly around $\left(\phi_{0}, 0\right)$, we expand $(\phi, \varphi)$ around this point to linear order, i.e., $k_{y} \approx$ $q_{0} \cos \phi_{0} \varphi$ and $p^{r}(\phi)^{*} p^{r}(\phi+\varphi) \approx \exp \left[2 i \eta_{0} \varphi /\left(\cos ^{2} \phi_{0}+\right.\right.$ $\left.\left.\eta_{0}^{2} \sin ^{2} \phi_{0}\right)\right]$. Using these, the integral in Eq. (D1) can be approximated by the standard Gaussian integral:

$$
F^{i, r}(y) \approx \varepsilon_{0}^{2} \int d \phi d \varphi e^{-\left[\left(\phi-\phi_{0}\right)^{2}+\left(\phi+\varphi-\phi_{0}\right)^{2}\right] /\left(2 \ell^{2}\right)-i \varphi Y^{i, r}(y)} \cos \varphi,
$$

where $Y^{i}(y)=q_{0} \cos \phi_{0} y$ and $Y^{r}(y)=q_{0} \cos \phi_{0} y-2 \eta_{0} /$ $\left(\cos ^{2} \phi_{0}+\eta_{0}^{2} \sin ^{2} \phi_{0}\right)$. Directly integrating Eq. (D3), the envelope functions can be written as

$$
\begin{aligned}
F^{i, r}(y) & \approx \pi \ell^{2} \varepsilon_{0}^{2}\left(e^{-\ell^{2}\left[Y^{i, r}(y)-1\right]^{2}}+e^{-\ell^{2}\left[Y^{i, r}(y)+1\right]^{2}}\right) \\
& =2 \pi \ell^{2} \varepsilon_{0}^{2} e^{-\left[\ell Y^{i, r}(y)\right]^{2}-\ell^{2}+O\left(\ell^{4}\right)}
\end{aligned}
$$

The center for $F^{i}(y)$ and $F^{r}(y)$ is therefore at $Y^{i, r}(y)=0$, yielding the wave packet centers at $x=0$ as

$$
y_{c}^{i}=0, \quad y_{c}^{r}=\frac{2 \eta_{0}}{q_{0}} \frac{\sec \phi_{0}}{\cos ^{2} \phi_{0}+\eta_{0}^{2} \sin ^{2} \phi_{0}},
$$

which gives the Hall shift as we discussed in the main text.

\section{APPENDIX E: MAGNETOPLASMON SCATTERING ACROSS A METALLIC INTERFACE}

In the main text, we analyzed reflection at a metalvacuum boundary. In this appendix, we discuss scattering of a plasmon wave at an interface between two uniform regions: region 1 and region 2 . The two regions have a sharp interface at $x=0$. They can have different $a_{0}$ and $\omega_{c}$. These are modeled by a step function: $a_{0}(x<0)=a_{0}^{(1)}$ and $a_{0}(x>0)=a_{0}^{(2)} ; \omega_{c}(x<0)=\omega_{c, 1}$ and $\omega_{c}(x>0)=\omega_{c, 2}$. For simplicity, we assume the incident wave is a monochromatic plane wave: $\mathcal{E}_{i}(\mathbf{r}, t)=\mathbf{E}_{i}\left(\mathbf{q}_{i}\right) \exp \left(i \omega_{\mathbf{q}_{i}} t-i \mathbf{q}_{i} \cdot \mathbf{r}\right)$, which excites the mode $\mathbf{u}\left(\mathbf{q}_{i}\right) \exp \left(i \omega_{\mathbf{q}_{i}} t-i \mathbf{q}_{i} \cdot \mathbf{r}\right)$. Current conservation at the $x=0$ interface requires

$$
p_{i} \mathbf{u}\left(\mathbf{q}_{i}\right) e^{i \omega_{\mathbf{q}_{i}} t}+p_{r} \mathbf{u}\left(\mathbf{q}_{r}\right) e^{i \omega_{\mathbf{q} r} t}=p_{t} \mathbf{u}\left(\mathbf{q}_{t}\right) e^{i \omega_{\mathbf{q}_{t}} t} .
$$

Here, $\mathbf{q}_{i}=\left(q_{1, x}, q_{y}\right), \mathbf{q}_{r}=\left(-q_{1, x}, q_{y}\right)$, and $\mathbf{q}_{t}=\left(q_{2, x}, q_{y}\right)$. $q_{y}$ is conserved because of translational symmetry in the $y$ direction. Applying energy conservation $a_{0}^{(1)}\left|\mathbf{q}_{r}\right|+\omega_{c, 1}^{2}=$ $a_{0}^{(2)}\left|\mathbf{q}_{t}\right|+\omega_{c, 2}^{2}$ yields $q_{1, x} \rightarrow-q_{1, x}$ for the reflected wave and $a_{0}^{(2)}\left(q_{2, x}^{2}+q_{y}^{2}\right)^{1 / 2}+\omega_{c, 2}^{2}=a_{0}^{(1)}\left(q_{1, x}^{2}+q_{y}^{2}\right)^{1 / 2}+\omega_{c, 1}^{2}$ for the transmitted wave.

Substituting the eigenmode

$$
\mathbf{u}_{1,2}(\mathbf{q})=\frac{\mathcal{N}_{1,2}}{q}\left(\begin{array}{l}
-i q_{x}+\eta_{1,2} q_{y} \\
-i q_{y}-\eta_{1,2} q_{x}
\end{array}\right)
$$

for the two regions, respectively, as well as the incident angle $\phi_{1}$, reflection angle $\pi-\phi_{1}$, transmission angle $\phi_{2}$, and the corresponding $\eta_{1,2}$ into the boundary condition Eq. (E1), we have

$$
\begin{aligned}
\frac{p_{r}}{p_{i}} & =\frac{-i\left(\eta_{1}-\eta_{2}\right) \cos \left(\phi_{1}-\phi_{2}\right)+\left(1-\eta_{1} \eta_{2}\right) \sin \left(\phi_{1}-\phi_{2}\right)}{-i\left(\eta_{1}-\eta_{2}\right) \cos \left(\phi_{1}+\phi_{2}\right)+\left(\eta_{1} \eta_{2}-1\right) \sin \left(\phi_{1}+\phi_{2}\right)}, \\
\frac{\mathcal{N}_{2} p_{t}}{\mathcal{N}_{1} p_{i}} & =\frac{\left(\eta_{1}^{2}-1\right) \sin 2 \phi_{1}}{-i\left(\eta_{1}-\eta_{2}\right) \cos \left(\phi_{1}+\phi_{2}\right)+\left(\eta_{1} \eta_{2}-1\right) \sin \left(\phi_{1}+\phi_{2}\right)},
\end{aligned}
$$


where $\mathcal{N}_{1}$ and $\mathcal{N}_{2}$ are normalization constants in regions 1 and 2 , respectively. When $\eta_{1}=\eta_{2}$, this gives the Fresnel equations:

$$
\frac{p_{r}}{p_{i}}=-\frac{\sin \left(\phi_{1}-\phi_{2}\right)}{\sin \left(\phi_{1}+\phi_{2}\right)}, \quad \frac{p_{t}}{p_{i}}=\frac{\sin 2 \phi_{1}}{\sin \left(\phi_{1}+\phi_{2}\right)} .
$$

When $\eta_{1} \neq \eta_{2}$, there are deviations from the conventional Fresnel equations [see Eq. (E3)]. This is because the current density textures in region 1 and region 2 are not perfectly matched.

\section{APPENDIX F: WAVE PACKET CENTER FOR CURRENT AND POWER DENSITY}

In the main text, we illustrate the plasmon Hall shift by considering wave packets of electric field intensity. As we now discuss, wave packets of other quantities can also experience plasmon Hall shifts. For example, the current density of the wave packet along its propagation direction can be written as

$$
\begin{aligned}
J^{i, r}(\mathbf{r})= & \operatorname{Re} \int d \mathbf{q} f(\mathbf{q}) p^{i, r}(\mathbf{q}) \mathbf{u}^{i, r}(\mathbf{q}) \cdot\left(\mathbf{q}^{i, r} / q\right) \\
& \times \exp \left(\mp i q_{x} x-i q_{y} y\right) \\
= & \operatorname{Re} \int d \mathbf{q} \mathcal{N}|f(\mathbf{q})| \exp \left[i \alpha^{i, r}(\mathbf{q})\right]
\end{aligned}
$$

where $\left(\mathbf{q}^{i, r} / q\right)$ is the unit vector along the propagating direction of mode $\mathbf{u}^{i, r}(\mathbf{q}) \exp \left(\mp i q_{x} x-i q_{y} y\right)$, and

$$
\begin{aligned}
& \alpha^{i}(\mathbf{q})=\delta(\mathbf{q})-q_{x} x-q_{y} y-\pi / 2, \\
& \alpha^{r}(\mathbf{q})=\delta(\mathbf{q})+q_{x} x-q_{y} y+\rho(\mathbf{q})+\pi / 2 .
\end{aligned}
$$

We find that the center of the current density (wave packet) is the same as $y_{c}^{i, r}(x)$ determined in Eq. (14) of the main text. To see this, note that the center of the wave packet can be determined by applying the method of stationary phases (see main text and Appendix C) directly on Eq. (F1) as

$$
\left[d \alpha^{i, r}(\mathbf{q}) / d q_{y}\right]_{\mathbf{q}=\mathbf{q}_{0}}=0 .
$$

Solving Eq. (F3) reduces to the expression in Eq. (14) of the main text, allowing the current density (along the propagation direction) to also experience the same plasmon Hall shift [Eq. (15)] as discussed in the main text.

In the same fashion, we can write the wave packet for the power density $\mathcal{I}^{i, r}(\mathbf{r})=\mathbf{E}^{i, r}(\mathbf{r}) \cdot \mathbf{J}^{i, r}(\mathbf{r})$ as

$$
\mathcal{I}^{i, r}(\mathbf{r})=\operatorname{Re} \int d \mathbf{q} d \mathbf{k} f_{\mathcal{I}}(\mathbf{q}, \mathbf{k}) \exp \left[i \theta^{i, r}(\mathbf{q}, \mathbf{k}, \mathbf{r})\right],
$$

where $f_{\mathcal{I}}(\mathbf{q}, \mathbf{k})=2 \pi \mathcal{N}^{2} q_{0}^{2}|f(\mathbf{q})||f(\mathbf{q}+\mathbf{k})| \cos \varphi / \omega_{q}$, with $\varphi$ the angle between $\mathbf{q}$ and $\mathbf{q}+\mathbf{k}$ for a monochromatic frequency. This gives a sharp peak for $f_{\mathcal{I}}(\mathbf{q}, \mathbf{k})$ at $\left(\mathbf{q}=\mathbf{q}_{0}, \mathbf{k}=0\right)$. The center of $\mathcal{I}^{i, r}(\mathbf{r})$ in real space is again the same saddle point $y_{c}^{i, r}(x)$ determined by Eq. (C3). This means that the power density also experiences the same plasmon Hall shift as discussed in Eq. (15) of the main text.

\section{APPENDIX G: STATIONARY PHASE FOR MULTIPLE FREQUENCIES}

In the main text, we concentrate on plasmon beams with a single frequency. This can be achieved by exciting plasmons via a laser beam with narrow linewidth; these sources are to all intents and purposes practically monochromatic. Nevertheless, we note parenthetically that the analysis in Appendix $\mathrm{C}$ can be extended to cases when the frequency has a distribution with a single peak. We first consider cases in which $\omega_{q}$ can take only discrete values: $\omega_{q} \in\left\{\omega_{n} \mid n \in \mathbb{N}\right\}$, with $\omega_{0}=\omega\left(q_{0}\right)$ having the largest amplitude. Focusing on the maximum amplitude $\omega_{0}$ distribution, we can follow the same analysis as in Appendix C to track the trajectory of the plasmon beam as in Eq. (14) of the main text.

For a continuous distribution $f(\mathbf{q})$ over multiple frequencies, a more elaborate analysis taking into account the distribution's shape and principal directions [42] can be employed. Finite width (in frequency space) corrections to quantities such as $\kappa_{0}$ occur only when the frequency distribution is correlated with its angle distribution $\phi$, and are typically small.

\section{APPENDIX H: PLASMON LOSS AND DECAY}

In the presence of disorder or phonon scattering, plasmon oscillations are damped over a finite plasmon lifetime $\tau$. This damping is parametrized by a quality factor $Q=\omega_{q} \tau / 2$, which measures the number of oscillations the plasmon experiences before it decays. Under ambient conditions, plasmons in typical graphene samples can have $\tau \sim 100 \mathrm{fs}$ and quality factors $Q \sim 8[14,15]$. Recently, ultrahigh-quality graphene encapsulated in $\mathrm{hBN}$ has been realized [20], possessing plasmons with $\tau \gtrsim 500 \mathrm{fs}$ and quality factors $Q \gtrsim 40$ at ambient temperatures.

In order to observe the plasmon Hall shift, high-quality samples with quality factor $Q \geq \Delta y_{H} / \lambda$ are needed; i.e., the plasmons have to at least experience $\Delta y_{H} / \lambda$ cycles before they decay. We note that for the range of plasmon Hall shifts shown in Fig. 3, quality factors found in readily available graphene samples $[14,15,20]$ are already sufficiently large to allow their observation.

Next, we estimate the magnetic fields required to observe the plasmon Hall shift. As shown in Fig. 3 and from Eq. (16), the largest Hall shifts occur when $\omega_{c} / \omega=$ $\cot \phi_{0}<1$, where the incident angles $\phi_{0}>\pi / 4$. As a specific example, we take $\phi=5 \pi / 12$ [red curve in Fig. 3(c) of the main text]. For this incident angle, the 
maximum Hall shift is $\Delta y_{H}=2.46 \lambda$ and occurs at $\omega_{c} / \omega_{q}=0.268$. For graphene samples, we estimate that this peak Hall shift can be readily measured at a realistic magnetic field around $2.37 \mathrm{~T}$ [at carrier density $n=10^{12} \mathrm{~cm}^{-2}$, Fermi velocity $v_{F}=10^{8} \mathrm{~cm} \mathrm{~s}^{-1}$, and plasmon frequency $\hbar \omega_{q}=50 \mathrm{meV}$ ]. For larger incident angles, even lower magnetic fields can be used (as long as $\left.Q \geq \Delta y_{H} / \lambda\right)$.

At low temperatures quality factors can be huge. For instance, $Q \sim 120$ have been reported at $T=60 \mathrm{~K}$ [21]. These samples in principle enable the Hall shifts that we predict to be measured with very low magnetic fields, and at very large incident angles.

We note, parenthetically, that the ability to access large incident angles is limited by the size and width of the plasmon beam; this is physically limited by the size and position of the beam launcher (e.g., size of nanoantenna [34]) fixed on the sample. While in principle very large incident angles enable giant plasmon Hall shifts (at small magnetic fields), this too is limited by the quality of the sample imposing an ultimate limit on the observable plasmon Hall shifts $\max \left(\Delta y_{H}\right) \leq Q \lambda$.

[1] C. L. Kane and E. J. Mele, Quantum Spin Hall Effect in Graphene, Phys. Rev. Lett. 95, 226801 (2005).

[2] M. König, S. Wiedmann, C. Brüne, A. Roth, H. Buhmann, L. W. Molenkamp, X. L. Qi, and S. C. Zhang, Quantum Spin Hall Insulator State in HgTe Quantum Wells, Science 318, 766 (2007).

[3] D. Xiao, W. Yao, and Q. Niu, Valley-Contrasting Physics in Graphene: Magnetic Moment and Topological Transport, Phys. Rev. Lett. 99, 236809 (2007).

[4] X. D. Xu, W. Yao, D. Xiao, and T. F. Heinz, Spin and Pseudospins in Layered Transition Metal Dichalcogenides, Nat. Phys. 10, 343 (2014).

[5] M. Onoda, S. Murakami, and N. Nagaosa, Hall Effect of Light, Phys. Rev. Lett. 93, 083901 (2004).

[6] K. Y. Bliokh and Y. P. Bliokh, Topological Spin Transport of Photons: The Optical Magnus Effect and Berry Phase, Phys. Lett. A 333, 181 (2004).

[7] F. D. M. Haldane and S. Raghu, Possible Realization of Directional Optical Waveguides in Photonic Crystals with Broken Time-Reversal Symmetry, Phys. Rev. Lett. 100, 013904 (2008).

[8] Z. Wang, Y. D. Chong, J. D. Joannopoulos, and M. Soljačić, Observation of Unidirectional Backscattering-Immune Topological Electromagnetic States, Nature (London) 461, 772 (2009).

[9] M. C. Chang and Q. Niu, Berry Phase, Hyperorbits, and the Hofstadter Spectrum, Phys. Rev. Lett. 75, 1348 (1995).

[10] D. Xiao, M. C. Chang, and Q. Niu, Berry Phase Effects on Electronic Properties, Rev. Mod. Phys. 82, 1959 (2010).

[11] C. W. J. Beenakker, R. A. Sepkhanov, A. R. Akhmerov, and J. Tworzydło, Quantum Goos-Hanchen Effect in Graphene, Phys. Rev. Lett. 102, 146804 (2009).
[12] K. Y. Bliokh, D. Smirnova, and F. Nori, Quantum Spin Hall Effect of Light, Science 348, 1448 (2015).

[13] M. Onoda, S. Murakami, and N. Nagaosa, Geometrical Aspects in Optical Wave-Packet Dynamics, Phys. Rev. E 74, 066610 (2006).

[14] J. N. Chen et al., Optical Nano-Imaging of Gate-Tunable Graphene Plasmons, Nature (London) 487, 77 (2012).

[15] Z. Fei et al., Gate-Tuning of Graphene Plasmons Revealed by Infrared Nano-Imaging, Nature (London) 487, 82 (2012).

[16] M. Jablan, M. Soljačić, and H. Buljan, Plasmons in Graphene: Fundamental Properties and Potential Applications, Proc. IEEE 101, 1689 (2013).

[17] F. H. L. Koppens, D. E. Chang, and F. J. García de Abajo, Graphene Plasmonics: A Platform for Strong Light-Matter Interactions, Nano Lett. 11, 3370 (2011).

[18] T. Low, A. Chaves, J. D. Caldwell, A. Kumar, N. X. Fang, P. Avouris, T. F. Heinz, F. Guinea, L. Martin-Moreno, and F. Koppens, Polaritons in Layered Two-Dimensional Materials, Nat. Mater. 16, 182 (2017).

[19] D. N. Basov, M. M. Fogler, and F. J. García de Abajo, Polaritons in van der Waals Materials, Science 354, aag1992 (2016).

[20] A. Woessner et al., Highly Confined Low-Loss Plasmons in Graphene-Boron Nitride Heterostructures, Nat. Mater. 14, 421 (2015).

[21] D. N. Basov, in Proceedings of the Graphene 2017 Conference, Barcelona (2017).

[22] K. Y. Bliokh, F. J. Rodriguez-Fortuno, F. Nori, and A. V. Zayats, Spin-Orbit Interactions of Light, Nat. Photonics 9 , 796 (2015).

[23] J. C. W. Song and M. S. Rudner, Chiral Plasmons without Magnetic Field, Proc. Natl. Acad. Sci. U.S.A. 113, 4658 (2016).

[24] A. Kumar, A. Nemilentsau, K. H. Fung, G. Hanson, N. X. Fang, and T. Low, Chiral Plasmon in Gapped Dirac Systems, Phys. Rev. B 93, 041413 (2016).

[25] A. Principi, M. I. Katsnelson, and G. Vignale, Edge Plasmons in Two-Component Electron Liquids in the Presence of Pseudomagnetic Fields, Phys. Rev. Lett. 117, 196803 (2016).

[26] D. F. Jin, L. Lu, Z Wang, C. Fang, J. D. Joannopoulos, M. Soljačić, L. Fu, and N.X. Fang, Topological Magnetoplasmon, Nat. Commun. 7, 13486 (2016).

[27] D. F. Jin, T. Christensen, M. Soljačić, N. X. Fang, L. Lu, and X. Zhang, Infrared Topological Plasmons in Graphene, Phys. Rev. Lett. 118, 245301 (2017).

[28] Z. F. Yu, G. Veronis, Z. Wang, and S. H. Fan, One-Way Electromagnetic Waveguide Formed at the Interface between a Plasmonic Metal under a Static Magnetic Field and a Photonic Crystal, Phys. Rev. Lett. 100, 023902 (2008).

[29] D. S. Borgnia, T. V. Phan, and L. S. Levitov, QuasiRelativistic Doppler Effect and Non-Reciprocal Plasmons in Graphene, arXiv:1512.09044.

[30] B. V. Duppen, A. Tomadin, A. N. Grigorenko, and M. Polini, Current-Induced Birefringent Absorption and NonReciprocal Plasmons in Graphene, 2D Mater. 3, 015011 (2016).

[31] K. Y. Bliokh, F. J. Rodríguez-Fortuño, A. Y. Bekshaev, Y.S. Kivshar, and F. Nori, Electric Current Induced 
Unidirectional Propagation of Surface Plasmon-Polaritons, Opt. Lett. 43, 963 (2018).

[32] K. W. Chiu and J. J. Quinn, Plasma Oscillations of a TwoDimensional Electron Gas in a Strong Magnetic Field, Phys. Rev. B 9, 4724 (1974); A. L. Fetter, Edge Magnetoplasmons in a Bounded Two-Dimensional Electron Fluid, Phys. Rev. B 32, 7676 (1985); K. I. Sasaki, S. Murakami, Y. Tokura, and H. Yamamoto, Determination of Intrinsic Lifetime of Edge Magnetoplasmons, Phys. Rev. B 93, 125402 (2016).

[33] The realness of $\mathcal{A}_{\hat{\mathbf{n}}}(\mathbf{q})$ can be discerned as follows. First, we note that $\nabla_{\mathbf{q}}\left[\left\langle u_{\hat{\mathbf{n}}}(\mathbf{q}) \mid u_{\hat{\mathbf{n}}}(\mathbf{q})\right\rangle\right]=\left[\left\langle u_{\hat{\mathbf{n}}}(\mathbf{q}) \mid \nabla_{\mathbf{q}} u_{\hat{\mathbf{n}}}(\mathbf{q})\right\rangle\right]^{*}+$ $\left\langle u_{\hat{\mathbf{n}}}(\mathbf{q}) \mid \nabla_{\mathbf{q}} u_{\hat{\mathbf{n}}}(\mathbf{q})\right\rangle$. Next, we note that since $\boldsymbol{\nabla}_{\mathbf{q}}\left[\left\langle u_{\hat{\mathbf{n}}}(\mathbf{q}) \mid u_{\hat{\mathbf{n}}}(\mathbf{q})\right\rangle\right]$ must also vanish, $\left\langle u_{\hat{\mathbf{n}}}(\mathbf{q}) \mid \nabla_{\mathbf{q}} u_{\hat{\mathbf{n}}}(\mathbf{q})\right\rangle$ must be pure imaginary. As a result, $\mathcal{A}_{\hat{\mathbf{n}}}(\mathbf{q})=\left\langle u_{\hat{\mathbf{n}}}(\mathbf{q})\left|i \boldsymbol{\nabla}_{\mathbf{q}}\right| u_{\hat{\mathbf{n}}}(\mathbf{q})\right\rangle$ is pure real. This is different from the typical Berry connection, which defines parallel transport in a local tangent plane spanned by $|\mathbf{u}(\mathbf{q})\rangle$. Here, $\mathcal{A}_{\hat{\mathbf{n}}}(\mathbf{q})$ is a geometric connection defining parallel transport projected to a fixed plane determined by the reflecting boundary.

[34] P. Alonso-González et al., Controlling Graphene Plasmons with Resonant Metal Antennas and Spatial Conductivity Patterns, Science 344, 1369 (2014).

[35] M. B. Lundeberg, Y. Gao, A. Woessner, C. Tan, P. AlonsoGonzález, K. Watanabe, T. Taniguchi, J. Hone, R. Hillenbrand, and F.H.L. Koppens, Thermoelectric
Detection and Imaging of Propagating Graphene Plasmons, Nat. Mater. 16, 204 (2017).

[36] G. Sundaram and Q. Niu, Wave-Packet Dynamics in Slowly Perturbed Crystals: Gradient Corrections and Berry-Phase Effects, Phys. Rev. B 59, 14915 (1999).

[37] S. C. Miller, Jr. and N. Ashby, Shifts of Electron Beam Position due to Total Reflection at a Barrier, Phys. Rev. Lett. 29, 740 (1972).

[38] D. M. Fradkin and R. J. Kashuba, Spatial Displacement of Electrons due to Multiple Total Reflections, Phys. Rev. D 9 , 2775 (1974).

[39] V. O. de Haan, J. Plomp, T. M. Rekveldt, W. H. Kraan, A. A. van Well, R. M. Dalgliesh, and S. Langridge, Observation of the Goos-Hänchen Shift with Neutrons, Phys. Rev. Lett. 104, 010401 (2010).

[40] Z. Wu, F. Zhai, F. M. Peeters, H. Q. Xu, and K. Chang, Valley-Dependent Brewster Angles and Goos-Hänchen Effect in Strained Graphene, Phys. Rev. Lett. 106, 176802 (2011).

[41] Q. D. Jiang, H. Jiang, H. W. Liu, Q. F. Sun, and X. C. Xie, Topological Imbert-Fedorov Shift in Weyl Semimetals, Phys. Rev. Lett. 115, 156602 (2015).

[42] A. Gray, E. Abbena, and S. Salamon, Modern Differential Geometry of Curves and Surfaces (CRC Press, Boca Raton, FL, 2006). 